\title{
Rosmarinic acid and arbutin suppress osteoclast differentiation by inhibiting superoxide and NFATc1 downregulation in RAW 264.7 cells
}

\author{
AKINA OMORI, YOSHITAKA YOSHIMURA, YOSHIAKI DEYAMA and KUNIAKI SUZUKI \\ Department of Molecular Cell Pharmacology, Hokkaido University Graduate School of Dental Medicine, \\ Sapporo, Hokkaido 060-8586, Japan
}

Received March 4, 2015; Accepted March 23, 2015

DOI: $10.3892 /$ br. 2015.452

\begin{abstract}
The present study investigated the effect of the natural polyphenols, rosmarinic acid and arbutin, on osteoclast differentiation in RAW 264.7 cells. Rosmarinic acid and arbutin suppressed osteoclast differentiation and had no cy totoxic effect on osteoclast precursor cells. Rosmarinic acid and arbutin inhibited superoxide production in a dose-dependent manner. mRNA expression of the master regulator of osteoclastogenesis, nuclear factor of activated $\mathrm{T}$ cells cytoplasmic 1 (NFATcl) and the osteoclast marker genes, matrix metalloproteinase-9, tartrate-resistant acid phosphatase and cathepsin-K, decreased following treatments with rosmarinic acid and arbutin. Furthermore, resorption activity decreased with the number of osteoclasts. These results suggest that rosmarinic acid and arbutin may be useful for the prevention and treatment of bone diseases, such as osteoporosis, through mechanisms involving inhibition of superoxide and downregulation of NFATCl.
\end{abstract}

Correspondence to: Dr Yoshitaka Yoshimura, Department of Molecular Cell Pharmacology, Hokkaido University Graduate School of Dental Medicine, Kita 13, Nishi 7, Sapporo, Hokkaido 060-8586, Japan

E-mail: yoshi@den.hokudai.ac.jp

Abbreviations: RAW cell, murine macrophage/osteoclast precursor cell line RAW 264.7 cell; RANKL, receptor activator of nuclear factor- $\kappa \mathrm{B}$ (RANK) ligand cells; ROS, reactive oxygen species; SOD, superoxide dismutase; NFATc1, nuclear factor of activated T cells cytoplasmic 1; TRAP, tartrate-resistant acid phosphatase; GAPDH, glyceraldehyde-3-phosphatedehydrogenase; FBS, fetal bovine serum; DMEM, Dulbecco's modified Eagle's medium; NF- $\kappa \mathrm{B}$, nuclear factor- $\kappa \mathrm{B}$

Key words: rosmarinic acid, arbutin, osteoclast, osteoclastogenesis, superoxide

\section{Introduction}

Polyphenols are aromatic and slightly bitter-tasting compounds in vegetables, fruits and herbs. Polyphenols have been reported to prevent several chronic diseases, such as arteriosclerosis, cardiovascular diseases and cancer, due to their antioxidant activity (1-3). The most well-known study associated with polyphenols is the investigation known as the French paradox. The French paradox is the epidemiological observation that the French population has a low incidence of coronary heart disease despite a diet high in saturated fat and is generally attributed to the high concentration of polyphenols in red wine $(4,5)$.

Previous studies have shown that polyphenols not only prevent arteriosclerosis and cancer, but also affect bone diseases by suppressing osteoclast differentiation and increasing bone mineral density in ovariectomized mice $(6,7)$. Several polyphenols, such as curcumin, resveratrol and plum polyphenol, can scavenge reactive oxygen species (ROS) associated with osteoclast differentiation (8-10). Rosmarinic acid is a natural polyphenol contained in Lamiaceae herbs, such as Perilla frutescens, lemon balm mint, sage and sweet basil (11). In particular, rosmarinic acid is effective against allergies, exhibits anti-inflammatory properties and is used in health foods $(12,13)$. Arbutin is a natural polyphenol present in plants such as Ericaceae, Asteraceae and Vaccinium (14). Arbutin has been reported to exert a potent inhibitory effect on the hydroxylation reaction of tyrosinase and has been widely used for skin whitening $(15,16)$.

Osteoclasts are members of the monocyte/macrophage lineage and are formed by fusion of their precursor cells. The receptor activator of nuclear factor $-\kappa \mathrm{B}$ (RANK) ligand (RANKL) is thought to be the most essential cytokine for the process of osteoclast differentiation and activation (17). RANKL is a member of the tumor necrosis factor (TNF) family and is expressed as a membrane-bound protein in osteoblast and stromal cells. Binding of RANKL with the receptor, RANK, induces activation of TNF receptor-associated factor 6 and the c-Fos pathway. This activates downstream signaling pathways involving the nuclear factor of activated T cells cytoplasmic 1 (NFATc1) and leads to the formation of tartrate-resistant acid phosphatase (TRAP)-positive osteoclasts (18). 
The murine macrophage/osteoclast precursor cell line, RAW 264.7 (RAW), is a widely used pre-osteoclast model. RAW cells differentiate into osteoclasts in the presence of RANKL. Bone is continuously and precisely remodeled by the coordination of bone-forming osteoblasts and bone-resorbing osteoclasts (19). Osteoporosis is characterized by reduced bone mass and diminished bone integrity caused by an imbalance between osteoblasts and osteoclasts (20).

ROS contribute to the aging process and the etiology of various degenerative diseases, including osteoporosis (21). Additionally, osteoclasts are activated by ROS, resulting in enhanced bone resorption. By contrast, ROS also play an important role as secondary messengers in osteoclast signaling pathways (22). ROS are generated by superoxide production of nicotinamide adenine dinucleotide phosphate oxidase in a process known as the respiratory burst. Approximately 3-10\% of the oxygen utilized by tissues is converted to ROS, including superoxides (23). A previous study reported that rosmarinic acid inhibited phorbol myristate acetate (PMA)-induced superoxide production in a macrophage cell line (24). Arbutin has been shown to inhibit ultraviolet A (UVA) irradiation-induced ROS in skin cells (25).

Rosmarinic acid has been shown to inhibit nuclear factor- $\mathrm{\kappa B}(\mathrm{NF}-\mathrm{\kappa B})$ activation during osteoclast formation (26). However, with the exception of this mechanism of action of rosmarinic acid, the inhibitory effects of polyphenols on osteoclasts remain unknown. In particular, the therapeutic effects of arbutin on bone have not been studied. The aim of the present study was to investigate the effects of rosmarinic acid and arbutin on the differentiation and formation of osteoclasts from RAW cells through suppression of the superoxide-mediated signaling pathway. We hypothesized that these polyphenols would downregulate $N F A T c 1$, resulting in the direct inhibition of osteoclastogenesis and lead to a decrease in osteoclast resorption activity.

\section{Materials and methods}

Chemicals. RAW cells were purchased from the American Type Culture Collection (Manassas, VA, USA). Rosmarinic acid, arbutin, Dulbecco's modified Eagle's medium (DMEM), $\alpha$-minimum essential medium ( $\alpha$-MEM), ethanol, Green Chemiluminescence $\mathrm{CD}$, methanol, trifluoroacetic acid and sodium hypochlorite were obtained from Wako Pure Chemical Industries, Ltd. (Osaka, Japan). Fetal bovine serum (FBS) and TRIzol were from Invitrogen Life Technologies (Carlsbad, CA, USA). Ascorbic acid 2-phosphate, L-alanyl-L-glutamine and Fast Red Violet LB salt were obtained from Sigma-Aldrich (St. Louis, MO, USA). RANKL was from Oriental Yeast Co., Ltd. (Tokyo, Japan).

Preparation of rosmarinic acid and arbutin. The structures of rosmarinic acid and arbutin are shown in Fig. 1. Rosmarinic acid was dissolved in ethanol. Arbutin was dissolved in deionized water. The reagents were sterilized by filtering through a $0.22-\mu \mathrm{m}$ membrane filter. Stock solutions of rosmarinic acid and arbutin were stored at $4^{\circ} \mathrm{C}$ until use.

Cell culture. The murine monocyte/macrophage cell line RAW was used as the osteoclast precursor cells. RAW cells differentiate into osteoclasts in the presence of RANKL $(50 \mathrm{ng} / \mathrm{ml})$. RAW cells were grown in DMEM supplemented with $10 \%$ heat-inactivated FBS and $66.7 \mu \mathrm{g} / \mathrm{ml}$ kanamycin-sulfate (Meiji Seika, Tokyo, Japan). The cells were seeded in 100-mm standard dishes (BD Biosciences, Franklin Lakes, NJ, USA). After overnight culture, the cells were cultured in $\alpha$-MEM supplemented with $10 \%$ heat-inactivated FBS, RANKL, $284 \mu \mathrm{M}$ ascorbic acid 2-phosphate, $2 \mathrm{mmol} / \mathrm{l}$ L-alanyl-L-glutamine and $66.7 \mu \mathrm{g} / \mathrm{ml}$ kanamycin-sulfate. The cells were subsequently treated with or without rosmarinic acid or arbutin at the concentrations indicated below. These cultures were fed every other day by replacing the old medium with an identical volume of fresh medium. All the cultures were incubated at $37^{\circ} \mathrm{C}$ in a humidified $5 \% \mathrm{CO}_{2}, 95 \%$ air atmosphere.

MTT assay for cell viability. A Vialight ${ }^{\mathrm{TM}}$ kit (Lonza, Allendale, NJ, USA) was used to measure the viability of RAW cells exposed to rosmarinic acid or arbutin. This kit utilizes the adenosine triphosphate present in live cells in a luciferase-catalyzed reaction to produce light. RAW cells were seeded in a 96-well plate (BD Biosciences) at a density of $0.25 \times 10^{4}$ cells/well and treated with rosmarinic acid or arbutin without RANKL for $72 \mathrm{~h}$. After culture, the cells were lysed in $50 \mu 1$ of cell lysis solution. After incubation for $15 \mathrm{~min}$ at room temperature, the cell lysates were transferred to a luminescence plate (Nunc A/S, Roskilde, Denmark) and mixed with $100 \mu \mathrm{l}$ AMR plus reagent. After 2 min incubation at room temperature in the dark, the plate was read on a luminescence plate reader (Wallac 1420 ARVOsx; PerkinElmer Inc., Waltham, MA, USA). The luminescence measured from each treatment group was converted to a percentage of the luminescence measured from the control cells (no rosmarinic acid or arbutin added).

TRAP staining. Osteoclasts were assessed by cytochemical staining for TRAP. RAW cells were seeded in a 48-well plate (BD Biosciences) at a density of $0.5 \times 10^{4}$ cells/well and treated with RANKL and rosmarinic acid or arbutin. After 6 days, the cells were fixed with $10 \%$ neutral formalin. Subsequently, the cells were washed with distilled water and stained in TRAP staining solution ( $\mathrm{pH}$ 5.0) with Fast Red Violet LB salt (27). TRAP-positive osteoclasts with $>2$ nuclei were considered to be osteoclasts. Osteoclasts with $\geq 8$ nuclei were considered to be large osteoclasts. The number of osteoclasts was counted under a light microscope.

Measurement of superoxide. Superoxide concentration was measured using a Green Chemiluminescence CD kit according to the manufacturer's instructions. Green Chemiluminescent $\mathrm{CD}$ is a highly sensitive chemiluminescence probe that reacts with the superoxide anion and a luminescence dye specific for the detection of superoxide. Briefly, $0.5 \times 10^{4}$ cells/well were seeded in a 48-well plate and treated with RANKL and rosmarinic acid or arbutin for $48 \mathrm{~h}$. The contents of the kit were dissolved in hot methanol:water 1:1 containing $0.1 \%$ (w/v) trifluoroacetic acid. To measure the superoxide produced in the medium, $200 \mu \mathrm{l}$ of the medium was mixed with $150 \mu \mathrm{l}$ of the reagent. The luminescence intensity of the samples was measured using a luminescence plate reader. The value 
A<smiles>O=C(C=Cc1ccc(O)c(O)c1)OC(Cc1ccc(O)c(O)c1)C(=O)O</smiles>

B

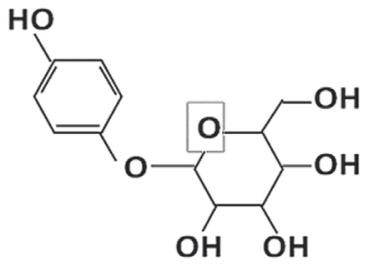

Figure 1. Structure of (A) rosmarinic acid and (B) arbutin.

for each treated group was converted to a percentage of the control luminescence.

Superoxide dismutase (SOD) activity. The SOD activity of the culture medium was measured using an SOD Assay kit-WST according to the manufacturer's instructions (Dojindo Molecular Technologies, Inc., Kumamoto, Japan). Briefly, the RAW cells were seeded in a 96-well plate at a density of $0.25 \times 10^{4}$ cells/well and treated with RANKL and rosmarinic acid or arbutin for $48 \mathrm{~h}$. The SOD activity was measured by mixing the reagents from $220 \mu \mathrm{l}$ of the WST kit with $20 \mu \mathrm{l}$ of the culture medium. After incubation for $20 \mathrm{~min}$ at $37^{\circ} \mathrm{C}$, absorbance was measured at $450 \mathrm{~nm}$ using a microplate reader (Bio-Rad Model 680; Bio-Rad, Hercules, CA, USA). The value for each treated group was converted to a percentage of the value obtained for the control group.

Quantitative polymerase chain reaction ( $q P C R$ ). RAW cells were seeded in a 6 -well plate at a density of $6 \times 10^{4}$ cells/well and treated with RANKL and rosmarinic acid or arbutin for $24 \mathrm{~h}$. After incubation, total RNA was isolated using TRIzol according to the manufacturer's instructions. cDNA synthesis was performed in $25 \mu \mathrm{l}$ from $1 \mu \mathrm{g}$ of total RNA using ReverTra Ace ${ }^{\circledR}$ reverse transcriptase (Toyobo Co., Ltd., Osaka, Japan) and oligo dT primers (Toyobo Co., Ltd.).

The specific primer sets used for NFATcl (Mm00479445 m1), matrix metalloproteinase-9(MMP-9) (Mm00442991_m1), TRAP (Mm00475698_m1), cathepsin-K (Mm00484036_m1) and glyceraldehyde-3-phosphate dehydrogenase (GAPDH) (Mm99999915_g1) were designed using the Primer Express program (Applied Biosystems, Foster City, CA, USA) and were purchased from Applied Biosystems. qPCR was performed using an ABI7300 (Applied Biosystems). The comparative threshold cycle $(\mathrm{Ct})$ method was used to calculate mRNA expression and the $\mathrm{Ct}$ values of the calibrator and the samples of interest were verified by normalizing to $G A P D H$. The comparative $\mathrm{Ct}$ method is also known as the $2^{-\Delta \Delta \mathrm{Ct}}$ method, where $\Delta \mathrm{Ct}=\Delta \mathrm{Ct}$ sample $-\Delta \mathrm{Ct}$ reference. $\Delta \mathrm{CT}$ sample is the $\mathrm{Ct}$ value for the sample normalized to the endogenous reference gene and $\Delta \mathrm{Ct}$ reference is the $\mathrm{Ct}$ value for the calibrator, which was also normalized to the endogenous reference gene.

Resorption pit assay. A pit formation assay was conducted using a Corning Osteo Assay Surface plate (Corning, Inc., Corning, NY, USA). RAW cells were seeded in plates at a density of $1 \times 10^{4}$ cells/well and treated with RANKL and rosmarinic acid or arbutin for 11 days. Cells were removed with $6 \%$ sodium hypochlorite solution. Subsequent to washing
A
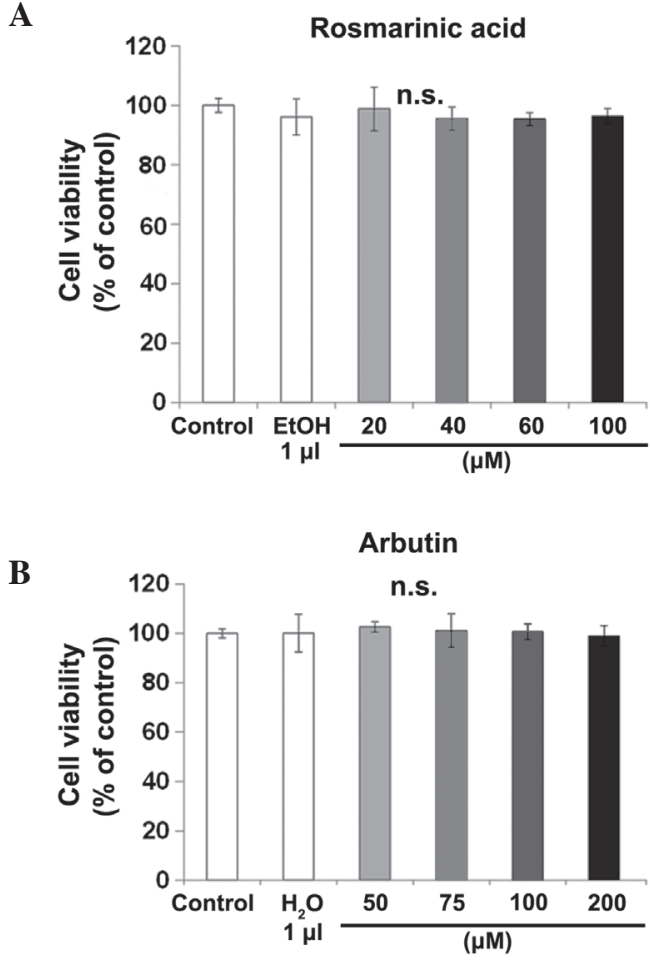

Figure 2. Effect of rosmarinic acid and arbutin on murine macrophage/osteoclast precursor cell line RAW 264.7 cell (RAW cell) viability. (A) RAW cells were treated with receptor activator of nuclear factor- $\kappa \mathrm{B}$ ligand and (B) rosmarinic acid or arbutin at the indicated concentrations for $72 \mathrm{~h}$. The luminescence intensity was determined. Data are representative of results from three separate experiments. Data are expressed as percentages of the value of the control cells (mean \pm standard deviation, $n=5$ ). n.s. not significant.

the wells with pure water and allowing them to dry, the wells were observed under a light microscope and the pit area was compared to the control.

Statistical analysis. All the data are presented as mean \pm standard deviation. Data were analyzed with the Dunnet test using StatView (version 5.0; SAS Institute Inc., Cary, NC, USA). Results with $\mathrm{P}<0.05$ and $\mathrm{P}<0.01$ were considered to indicate a statistically significant difference.

\section{Results}

Effect of rosmarinic acid and arbutin on cell viability in osteoclast precursor cells. In order to investigate the potential cytotoxicity of rosmarinic acid and arbutin on RAW cells as osteoclast precursors, cell viability was examined 


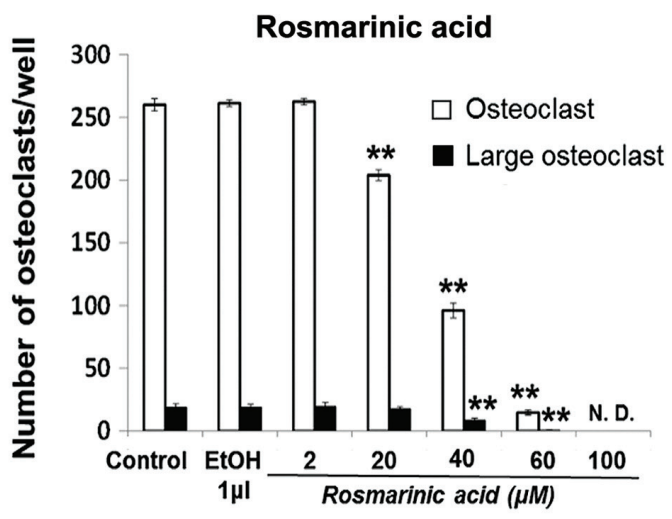

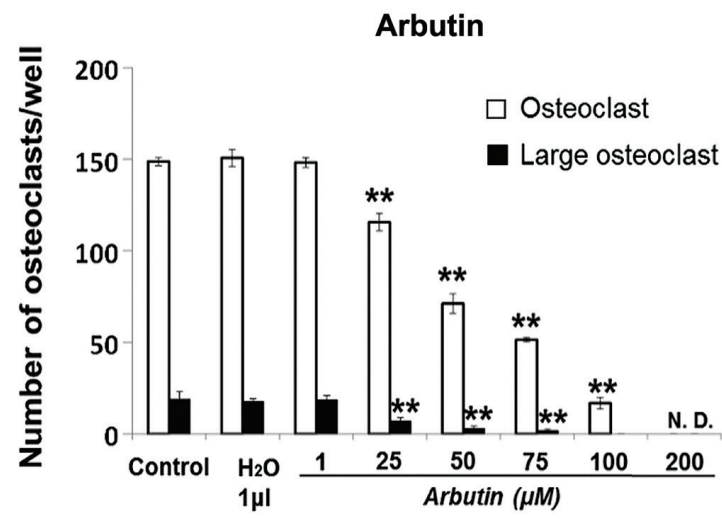

C

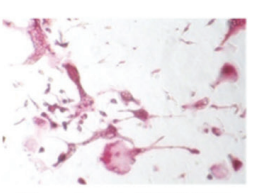

Control (EtOH)

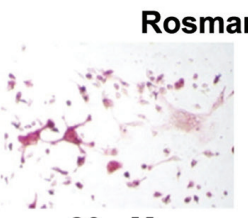

$20 \mu \mathrm{M}$

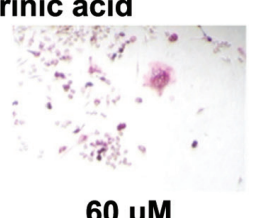

$60 \mu \mathrm{M}$

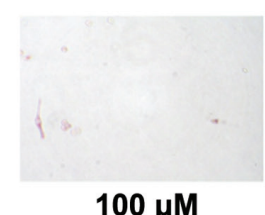

$100 \mu \mathrm{M}$

D

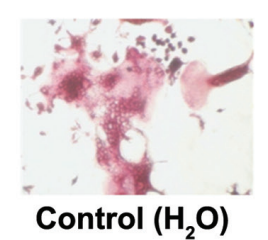

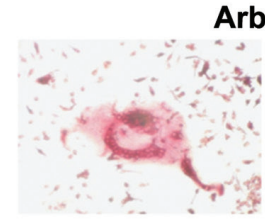

$25 \mu \mathrm{M}$
Arbutin

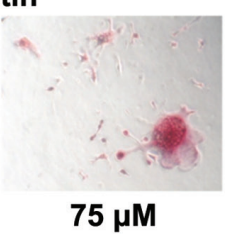

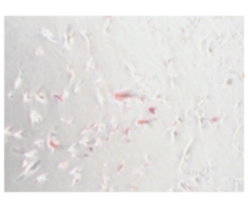

$200 \mu \mathrm{M}$

Figure 3. Inhibitory effect of rosmarinic acid and arbutin on receptor activator of nuclear factor- $\kappa \mathrm{B}$ ligand (RANKL)-induced osteoclasts from murine macrophage/osteoclast precursor cell line RAW 264.7 cells (RAW cells). RAW cells were treated with RANKL and rosmarinic acid or arbutin at the indicated concentrations. After 6 days in culture, the cells were fixed and stained for tartrate-resistant acid phosphatase (TRAP). (A and B) TRAP-positive multinucleated osteoclasts and TRAP-positive large multinucleated osteoclasts were counted under a microscope. (C and D) TRAP-positive osteoclasts were visualized by light microphotography. Data are representative of results from three separate experiments. Data are expressed as percentages of the value of the control cells (mean \pm standard deviation, $\mathrm{n}=5)$. $\left({ }^{* *} \mathrm{P}<0.01\right.$ vs. control).

using the MTT assay (Fig. 2). Raw cells were incubated with different concentrations of rosmarinic acid or arbutin for $72 \mathrm{~h}$. Rosmarinic acid at 20,40,60 and $100 \mu \mathrm{M}$ (Fig. 2A) and arbutin at 50, 75, 100 and $200 \mu \mathrm{M}$ (Fig. 2B) did not affect growth, as compared to the control cells. The viability of the cells at all the concentrations tested was $>95 \%$.

Rosmarinic acid and arbutin inhibits RANKL-induced osteoclast differentiation. Osteoclast differentiation was dose-dependently inhibited by rosmarinic acid and arbutin (Fig. 3A and B), as shown in Fig. 3C and D. The presence of rosmarinic acid at 20,40,60 and $100 \mu \mathrm{M}$ decreased the number of TRAP-positive cells to $78,37,6$ and $0 \%$ of the control level, respectively (Fig. 3A). The presence of arbutin at 25, 50, 75,100 and $200 \mu \mathrm{M}$ decreased the number of TRAP-positive osteoclasts to $\sim 78,48,35,11$ and $0 \%$ of the control level, respectively (Fig. 3B). The number of TRAP-positive large osteoclasts decreased with increasing concentrations of rosmarinic acid (Fig. 3C) and arbutin (Fig. 3D).

Rosmarinic acid and arbutin inhibits superoxide production and SOD activity. The effect of rosmarinic acid or arbutin on superoxide production (Fig. 4A and B) was investigated in osteoclasts. RAW cells were incubated with rosmarinic acid $(20,40$ and $60 \mu \mathrm{M})$ or arbutin $(50,75$ and $100 \mu \mathrm{M})$ for $48 \mathrm{~h}$.
Rosmarinic acid and arbutin decreased superoxide production in a dose-dependent manner. By contrast, all the concentrations of rosmarinic acid and arbutin tested had no effect on SOD inhibition (Fig. 4C and D).

Effect of rosmarinic acid and arbutin on $m R N A$ expression of osteoclastic-specific genes. Rosmarinic acid and arbutin suppressed RANKL-induced expression of osteoclastogenesis in a dose-dependent manner after $24 \mathrm{~h}$ (Fig. 5).

Rosmarinic acid and arbutin inhibits $R A N K L$-induced resorption by the pit area. Rosmarinic acid and arbutin decreased resorption by the pit area in a dose-dependent manner (Fig. 6A and B). Resorption by the pit area decreased in line with the number of osteoclasts, suggesting that rosmarinic acid and arbutin primarily affect osteoclast differentiation as opposed to activity.

\section{Discussion}

The present study reported that rosmarinic acid and arbutin at non-toxic concentrations suppress the number of TRAP-positive osteoclasts and large osteoclasts from RAW cells. The results clearly suggest that rosmarinic acid and arbutin suppress osteoclast formation by directly acting 
A

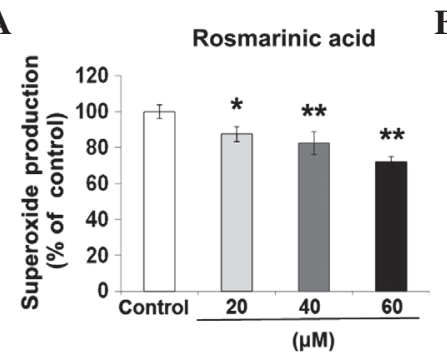

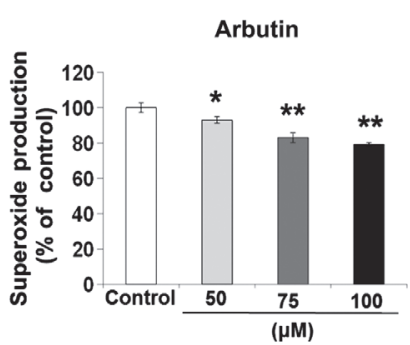

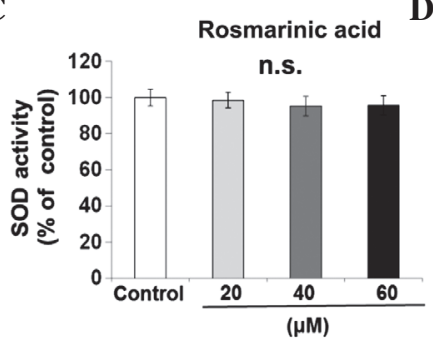

D

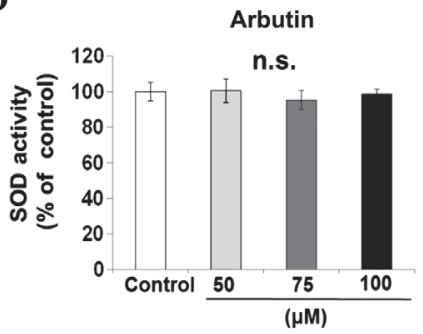

Figure 4. Effect of rosmarinic acid and arbutin on superoxide production and superoxide dismutase (SOD) activity. Murine macrophage/osteoclast precursor cell line RAW 264.7 cells were cultured with receptor activator of nuclear factor- $\mathrm{\kappa B}$ ligand and the indicated concentration of rosmarinic acid or arbutin for $48 \mathrm{~h}$. (A and B) Superoxide production was measured by a luminescence intensity assay using a luminescence plate reader. (C and D) SOD activity was measured at $450 \mathrm{~nm}$ using a microplate reader. Data are representative of results from four separate experiments. Data are expressed as percentages of the value of the control cells (mean \pm standard deviation, $n=5)$. ("P<0.05, ${ }^{* *} \mathrm{P}<0.01$, vs control). n.s., not significant.

A

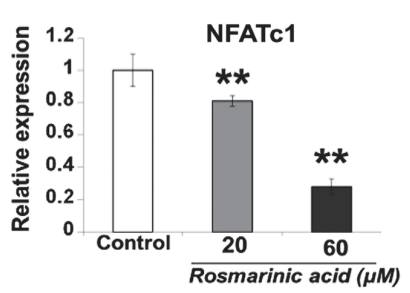

$\mathbf{E}$

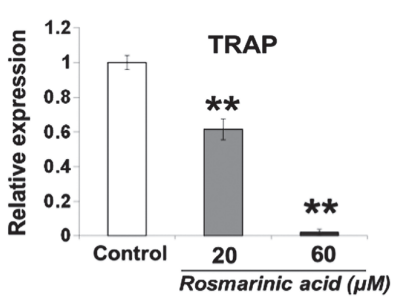

B

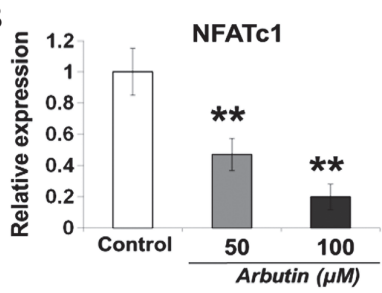

F

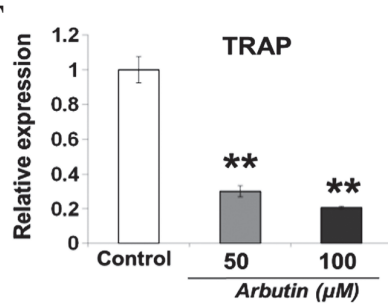

C

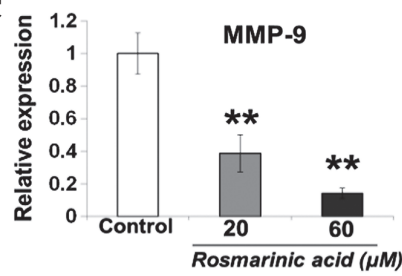

G

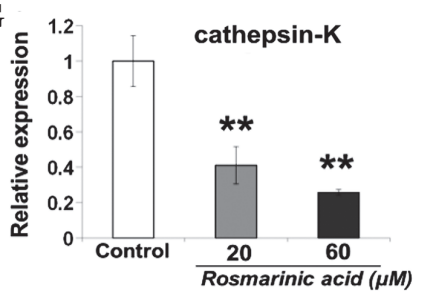

D

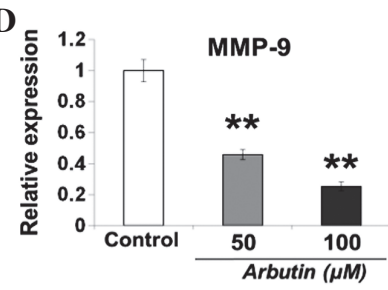

$\mathbf{H}$

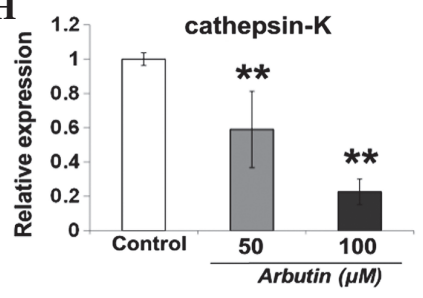

Figure 5. Expression of mRNA for the master regulator of osteoclastogenesis, nuclear factor of activated T cells cytoplasmic 1 (NFATc1) and the osteoclast-specific marker genes, matrix metalloproteinase-9 (MMP-9), tartrate-resistant acid phosphatase (TRAP) and cathepsin-K, by quantitative polymerase chain reaction (qPCR). Murine macrophage/osteoclast precursor cell line RAW 264.7 cells were seeded in a 6-well plate at a density of 6x10 4 cells/well and treated with receptor activator of nuclear factor- $\mathrm{KB}$ ligand and rosmarinic acid or arbutin at the indicated concentration for $24 \mathrm{~h}$. (A and B) NFATcl, (C and D) MMP-9, (E and F) TRAP and ( $\mathrm{G}$ and $\mathrm{H}$ ) cathepsin-K expression was analyzed by qPCR as described in the Materials and methods. Results are shown as fold-change or relative quantitation of target expression $\left(2^{-\triangle \Delta \mathrm{Ct}}\right.$ method) relative to the control following normalization against GAPDH expression. Data are representative of results from four separate experiments. Data are expressed as percentages of the value of the control cells (mean \pm standard deviation, $\mathrm{n}=5$ ). $($ (*) $\mathrm{P}<0.01$ vs. control).

A

\section{Rosmarinic acid}

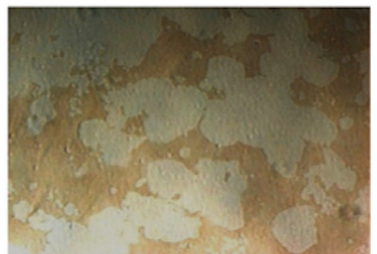

Control (EtOH)

B

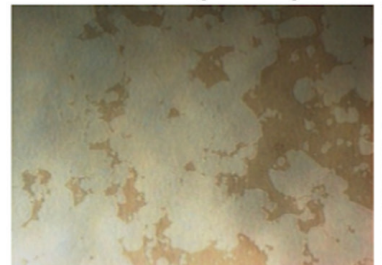

Control $\left(\mathrm{H}_{2} \mathrm{O}\right)$

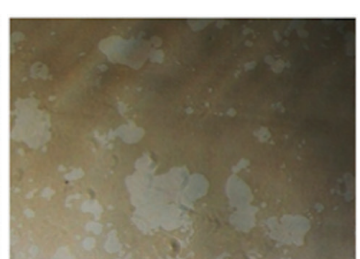

$20 \mu \mathrm{M}$

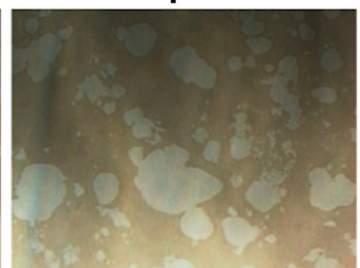

$25 \mu \mathrm{M}$

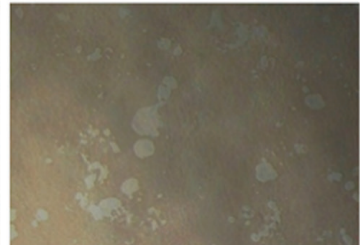

$60 \mu \mathrm{M}$

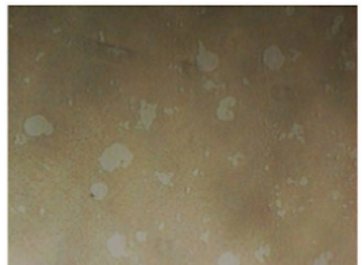

$75 \mu \mathrm{M}$

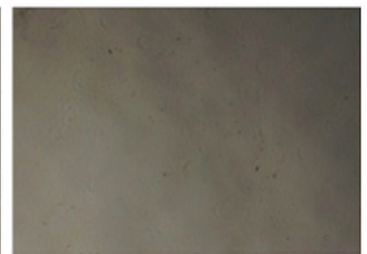

$100 \mu \mathrm{M}$

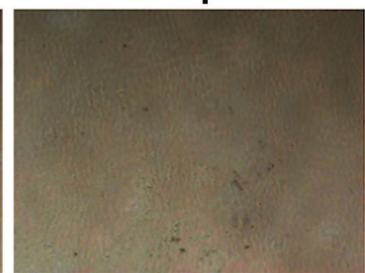

$200 \mu \mathrm{M}$

Figure 6. Inhibitory effect of rosmarinic acid and arbutin on receptor activator of nuclear factor- $\mathrm{B}$ ligand (RANKL)-induced resorption pit area. Murine macrophage/osteoclast precursor cell line RAW 264.7 cells were seeded in a 24 -well plate at a density of $1 \times 10^{4}$ cells/well and treated with RANKL and (A) rosmarinic acid or (B) arbutin at the indicated concentration. After 11 days culture, the cells were washed with phosphate-buffered saline and removed from the well walls with $6 \%$ sodium hypochlorite solution. Images of the resorption pit area were captured. Data are representative of results from three separate experiments $(\mathrm{n}=3)$. 
on osteoclast precursor cells. Rosmarinic acid has previously been reported to suppress osteoclast differentiation of murine bone marrow-derived macrophages (BMM) and RAW cells (26). However, the regulation by rosmarinic acid of osteoclast differentiation needs to be further defined. Arbutin has not previously been reported to inhibit osteoclast differentiation or to show a beneficial effect on bone. To the best of our knowledge, the study reports for the first time that arbutin inhibits osteoclast differentiation of RAW cells. In particular, the inhibitory effects of rosmarinic acid at a $20 \mu \mathrm{M}$ concentration is similar to the inhibitory effect of arbutin at $25 \mu \mathrm{M}$ : $21.54 \%$ caused by $20 \mu \mathrm{M}$ rosmarinic acid and $22.19 \%$ caused by $25 \mu \mathrm{M}$ arbutin. Furthermore, rosmarinic acid has been reported as more potent for ROS removal compared to arbutin in the mesenteric artery in rats (28). Therefore, whether the antioxidant reaction of rosmarinic acid and arbutin can regulate osteoclast differentiation was investigated.

ROS was recognized as a secondary messenger in the differentiation of osteoclasts and plays an important role in differentiation (22). By contrast, excessive ROS production results in an abnormal osteoclast phenomenon: The activation of osteoclast signals due to an increase in the number of osteoclasts (29). Additionally, ROS production by osteoporotic bone tissue is significantly higher compared to normal bone tissue (30). Several studies have reported that the ROS level increases during RANKL-induced osteoclast differentiation and that this ROS generation can be attenuated by polyphenols such as curcumin and resveratrol $(8,9)$. In addition, the antioxidants scopoletin and scopolin inhibit ROS and superoxide production in a dose-dependent manner (31). A superoxide is converted into various ROS, such as hydroxyl radical, hydrogen peroxide and peroxynitrite, in the process of osteoclast formation $(32,33)$. Thus, to investigate whether rosmarinic acid and arbutin can inhibit superoxide generation during osteoclast differentiation, the production of superoxide was analyzed. According to the sucopoletin and scopolin study referred to above, the ROS level increased to its highest value within $48 \mathrm{~h}$ and subsequently decreased (31). Therefore, the superoxide levels were also analyzed after treating the cells with rosmarinic acid and arbutin for $48 \mathrm{~h}$ and showed that rosmarinic acid and arbutin reduced superoxide production in a dose-dependent manner. Of note, rosmarinic acid and arbutin inhibited superoxide, but had no effect on RANKL-induced SOD activity, suggesting rosmarinic acid and arbutin have a positive effect on RANKL-induced superoxide removal. The antioxidant activity of rosmarinic acid and arbutin have been observed using various cells. Rosmarinic acid inhibits ROS production in several cell lines, such as dopaminergic cells and hematoma cells $(34,35)$. Rosmarinic acid also inhibits PMA-induced intracellular superoxide in macrophage cell lines and has no effect on copper/zinc SOD (24). Arbutin suppresses UVA-induced ROS on cellular tyrosinase activity, primarily in skin cells, such as human and mice melanocytes $(25,36)$. Additionally, curcumin suppresses phosphor I- $\kappa \mathrm{B} \alpha$ and intracellular ROS production in the osteoclast differentiation process in a dose-dependent manner (8). Curcumin also completely suppresses NF- $\kappa \mathrm{B}$ activity in the osteoclast differentiation process (37). Rosmarinic acid shares common structural features with curcumin, so we can speculate that rosmarinic acid acts by a similar mechanism to inhibit
RANKL-induced production of ROS, such as superoxide. Furthermore, it was previously demonstrated that rosmarinic acid inhibits RANKL-induced NF- $\kappa \mathrm{B}$ activation in the osteoclast differentiation process in BMM (26). Arbutin suppresses $\mathrm{NF}-\kappa \mathrm{B}$ activity in BV2 microglial cells (38). Further investigation is required to ascertain whether inhibition of $N F-\kappa B$ activity plays a role in suppression of osteoclast differentiation from RAW cells by rosmarinic acid and arbutin.

Taken together, the present results show that rosmarinic acid and arbutin decrease RANKL-induced expression of NFATcl mRNA. NFATcl is a master regulator of RANKL-induced osteoclast differentiation (39). NFATcl plays a pivotal role in osteoclast activation via upregulation of various genes in a series of processes, such as osteoclast adhesion, migration, acidification and degradation of inorganic and organic bone matrix (18). Thus, the effect of rosmarinic acid and arbutin on the mRNA expression of the osteoclast marker genes, MMP-9, TRAP and cathepsin-K, was investigated in osteoclasts. The mRNA expression of $M M P-9, T R A P$ and cathepsin-K was also suppressed by the addition of rosmarinic acid and arbutin. MMP-9 is essential for initiating the osteoclastic resorption process by removing the collagenous layer from the bone surface prior to demineralization (40). Furthermore, expression of MMP-9 in osteoclast is markedly higher compared to other cell types (41). Tea polyphenols suppress osteoclast formation and activity by inhibiting the production of free radicals and MMP-9 and inducing apoptosis (42). TRAP is a marker for osteoclasts and there is increasing evidence of its proteolytic role in bone resorption (43). TRAP knockout mice have shown that bone shape and modeling are altered by increased mineral density, suggesting that TRAP plays an important role in bone resorption (44). Cathepsin-K activity is required for the initial formation of actin rings and thus for the activation of osteoclasts (45). An NFATc1 knockout murine study has reported that the endocardial expression of NFATCl is dependent on the expression of cathepsin-K (46). Based on these findings, we hypothesized that rosmarinic acid and arbutin may suppress the resorption activity of osteoclasts. To investigate the effect of resorption activity of osteoclasts treated with rosmarinic acid and arbutin, cells were treated with rosmarinic acid and arbutin after 11 days of culture using Corning Osteo Assay Surface plates. Pit assays compared the pit area following treatment with rosmarinic acid and arbutin to that of the controls, suggesting that rosmarinic acid and arbutin impair the resorption activity of osteoclasts.

It is critical to investigate the effect of compounds in vivo rather than in vitro. Increasing studies are therefore required to test the beneficial bone effects of rosmarinic acid and arbutin in animal models and human studies. From the viewpoint of clinical applications, it has been reported that the concentration of rosmarinic acid in the plasma of goat kids fed a diet containing 140.44 or $273.86 \mathrm{mg} / \mathrm{kg}$ rosmarinic acid for 224 days was 0.78 and $0.81 \mathrm{mg} / 1$, respectively (47). The present results suggest that such plasma concentrations can significantly inhibit osteoclasts. Rosmarinic acid, as well as other polyphenols such as caffeic acid and ferulic acid, is metabolized when taken orally (48). A rat serum concentration of $1.36 \mu \mathrm{mol} / \mathrm{min} / 1$ was obtained by dosing $100 \mu \mathrm{mol} / \mathrm{kg} / \mathrm{body}$ weight rosmarinic acid (49). These studies apparently show the effective concentration difference between in vitro and blood 
concentrations. The blood levels of arbutin have not been reported. However, arbutin is used as a traditional medicine for treating urinary tract infections, thereby confirming its safety when taken orally (50).

In conclusion, the effect of rosmarinic acid and arbutin on osteoclast differentiation was analyzed by studying their inhibition of RANKL-induced superoxide, which is the source of ROS generation. Rosmarinic acid and arbutin inhibited osteoclast formation by blocking osteoclast marker genes, such as $M M P-9, T R A P$ and cathepsin-K, via downregulation of NFATCl. These findings strongly suggest that rosmarinic acid and arbutin hold promise for the treatment of various bone diseases, such as osteoporosis and bone metastasis, associated with excessive bone resorption.

\section{References}

1. Aviram M and Fuhrman B: Polyphenolic flavonoids inhibit macrophage-mediated oxidation of LDL and attenuate atherogenesis. Atherosclerosis 137 (Suppl): S45-S50, 1998.

2. Basu A, Du M, Leyva MJ, Sanchez K, Betts NM, Wu M, Aston CE and Lyons TJ: Blueberries decrease cardiovascular risk factors in obese men and women with metabolic syndrome. J Nutr 140: 1582-1587, 2010.

3. Wang P, Heber D and Henning SM: Quercetin increased bioavailability and decreased methylation of green tea polyphenols in vitro and in vivo. Food Funct 3: 635-642, 2012.

4. Dudley JI, Lekli I, Mukherjee S, Das M, Bertelli AA and Das DK: Does white wine qualify for French paradox? Comparison of the cardioprotective effects of red and white wines and their constituents: Resveratrol, tyrosol and hydroxytyrosol. J Agric Food Chem 56: 9362-9373, 2008.

5. Vidavalur R, Otani H, Singal PK and Maulik N: Significance of wine and resveratrol in cardiovascular disease: French paradox revisited. Exp Clin Cardiol 11: 217-225, 2006.

6. Ko CH, Lau KM, Choy WY and Leung PC: Effects of tea catechins, epigallocatechin, gallocatechin and gallocatechin gallate, on bone metabolism. J Agric Food Chem 57: 7293-7297, 2009.

7. Hagiwara K, Goto T, Araki M, Miyazaki $\mathrm{H}$ and Hagiwara H: Olive polyphenol hydroxytyrosol prevents bone loss. Eur J Pharmacol 662: 78-84, 2011.

8. Moon HJ, Ko WK, Han SW, Kim DS, Hwang YS, Park HK and Kwon IK: Antioxidants, like coenzyme Q10, selenite and curcumin, inhibited osteoclast differentiation by suppressing reactive oxygen species generation. Biochem Biophys Res Commun 418: 247-253, 2012

9. He X, Andersson G, Lindgren U and Li Y: Resveratrol prevents RANKL-induced osteoclast differentiation of murine osteoclas progenitor RAW 264.7 cells through inhibition of ROS production. Biochem Biophys Res Commun 401: 356-362, 2010.

10. Bu SY, Lerner M, Stoecker BJ, Boldrin E, Brackett DJ, Lucas EA and Smith BJ: Dried plum polyphenols inhibit osteoclastogenesis by downregulating NFATc1 and inflammatory mediators. Calcif Tissue Int 82: 475-488, 2008.

11. Takano H, Osakabe N, Sanbongi C, Yanagisawa R, Inoue K, Yasuda A, Natsume M, Baba S, Ichiishi E and Yoshikawa T: Extract of Perilla frutescens enriched for rosmarinic acid, a polyphenolic phytochemical, inhibits seasonal allergic rhinoconjunctivitis in humans. Exp Biol Med (Maywood) 229: 247-254, 2004.

12. al-Sereiti MR, Abu-Amer KM and Sen P: Pharmacology of rosemary (Rosmarinus officinalis Linn.) and its therapeutic potentials. Indian J Exp Biol 37: 124-130, 1999.

13. Oh HA, Park CS, Ahn HJ, Park YS and Kim HM: Effect of Perilla frutescens var. acuta Kudo and rosmarinic acid on allergic inflammatory reactions. Exp Biol Med (Maywood) 236: 99-106, 2011.

14. Pavlović RD, Lakusić B, Doslov-Kokorus Z and Kovacević N: Arbutin content and antioxidant activity of some Ericaceae species. Pharmazie 64: 656-659, 2009.

15. Maeda K and Fukuda M: Arbutin: Mechanism of its depigmenting action in human melanocyte culture. J Pharmacol Exp Ther 276: 765-769, 1996.
16. Sugimoto K, Nishimura T, Nomura K, Sugimoto K and Kuriki T: Syntheses of arbutin-alpha-glycosides and a comparison of their inhibitory effects with those of alpha-arbutin and arbutin on human tyrosinase. Chem Pharm Bull (Tokyo) 51: 798-801, 2003.

17. Väänänen HK and Laitala-Leinonen T: Osteoclast lineage and function. Arch Biochem Biophys 473: 132-138, 2008.

18. Takayanagi H: The role of NFAT in osteoclast formation. Ann NY Acad Sci 1116: 227-237, 2007

19. Corral DA, Amling M, Priemel M, Loyer E, Fuchs S, Ducy P, Baron R and Karsenty G: Dissociation between bone resorption and bone formation in osteopenic transgenic mice. Proc Natl Acad Sci USA 95: 13835-13840, 1998.

20. Simon LS: Osteoporosis. Clin Geriatr Med 21: 603-629, 2005.

21. Manolagas SC: From estrogen-centric to aging and oxidative stress: A revised perspective of the pathogenesis of osteoporosis. Endocr Rev 31: 266-300, 2010.

22. Sasaki H, Yamamoto H, Tominaga K, Masuda K, Kawai T, Teshima-Kondo S and Rokutan K: NADPH oxidase-derived reactive oxygen species are essential for differentiation of a mouse macrophage cell line (RAW264.7) into osteoclasts. J Med Invest 56: 33-41, 2009.

23. Vasilaki A, Mansouri A, Van Remmen H, van der Meulen JH, Larkin L, Richardson AG, McArdle A, Faulkner JA and Jackson MJ: Free radical generation by skeletal muscle of adult and old mice: Effect of contractile activity. Aging Cell 5: 109-117, 2006.

24. Qiao S, Li W, Tsubouchi R, Haneda M, Murakami K, Takeuchi F, Nisimoto $\mathrm{Y}$ and Yoshino $\mathrm{M}$ : Rosmarinic acid inhibits the formation of reactive oxygen and nitrogen species in RAW264.7 macrophages. Free Radic Res 39: 995-1003, 2005.

25. Xiao L, Matsubayashi K and Miwa N: Inhibitory effect of the water-soluble polymer-wrapped derivative of fullerene on UVA-induced melanogenesis via downregulation of tyrosinase expression in human melanocytes and skin tissues. Arch Dermatol Res 299: 245-257, 2007.

26. Hsu YC, Cheng CP and Chang DM: Plectranthus amboinicus attenuates inflammatory bone erosion in mice with collagen-induced arthritis by downregulation of RANKL-induced NFATc1 expression. J Rheumatol 38: 1844-1857, 2011.

27. Takeyama S, Yoshimura Y, Deyama Y, Sugawara Y, Fukuda H and Matsumoto A: Phosphate decreases osteoclastogenesis in coculture of osteoblast and bone marrow. Biochem Biophys Res Commun 282: 798-802, 2001.

28. Broskova Z, Drabikova K, Sotnikova R, Fialova S and Knezl V: Effect of plant polyphenols on ischemia reperfusion injury of the isolated rat heart and vessels. Phytother Res 27: 1018-1022, 2013.

29. Yang S, Zhang Y, Ries W and Key L: Expression of Nox4 in osteoclasts. J Cell Biochem 92: 238-248, 2004

30. Tang XL, Liu XJ, Tian Q and Zhang W: Dynamic oxidative stress and DNA damage induced by oestrogen deficiency and protective effects of puerarin and $17 \beta$-oestradiol in ovariectomized rats. Basic Clin Pharmacol Toxicol 111: 87-91, 2012.

31. Lee SH, Ding Y, Yan XT, Kim YH and Jang HD: Scopoletin and scopolin isolated from Artemisia iwayomogi suppress differentiation of osteoclastic macrophage RAW 264.7 cells by scavenging reactive oxygen species. J Nat Prod 76: 615-620, 2013.

32. Jones RD, Hancock JT and Morice AH: NADPH oxidase: A universal oxygen sensor? Free Radic Biol Med 29: 416-424, 2000.

33. Steinbeck MJ, Kim JK, Trudeau MJ, Hauschka PV and Karnovsky MJ: Involvement of hydrogen peroxide in the differentiation of clonal HD-11EM cells into osteoclast-like cells. J Cell Physiol 176: 574-587, 1998.

34. Lee HJ, Cho HS, Park E, Kim S, Lee SY, Kim CS, Kim K, Kim SJ and Chun HS: Rosmarinic acid protects human dopaminergic neuronal cells against hydrogen peroxide-induced apoptosis. Toxicology 250: 109-115, 2008.

35. Renzulli C, Galvano F, Pierdomenico L, Speroni E and Guerra MC: Effects of rosmarinic acid against aflatoxin B1 and ochratoxin-A-induced cell damage in a human hepatoma cell line (Hep G2). J Appl Toxicol 24: 289-296, 2004.

36. Hu ZM, Zhou Q, Lei TC, Ding SF and Xu SZ: Effects of hydroquinone and its glucoside derivatives on melanogenesis and antioxidation: Biosafety as skin whitening agents. J Dermatol Sci 55: 179-184, 2009

37. Bharti AC, Takada Y and Aggarwal BB: Curcumin (diferuloylmethane) inhibits receptor activator of NF-kappa B ligand-induced NF-kappa B activation in osteoclast precursors and suppresses osteoclastogenesis. J Immunol 172: 5940-5947, 2004. 
38. Lee HJ and Kim KW: Anti-inflammatory effects of arbutin in lipopolysaccharide-stimulated BV2 microglial cells. Inflamm Res 61: 817-825, 2012.

39. Asagiri M, Sato K, Usami T, Ochi S, Nishina H, Yoshida H, Morita I, Wagner EF, Mak TW, Serfling E, et al: Autoamplification of NFATc1 expression determines its essential role in bone homeostasis. J Exp Med 202: 1261-1269, 2005.

40. Delaissé JM, Engsig MT, Everts V, del Carmen Ovejero M, Ferreras M, Lund L, Vu TH, Werb Z, Winding B, Lochter A, et al: Proteinases in bone resorption: Obvious and less obvious roles. Clin Chim Acta 291: 223-234, 2000.

41. Tezuka K, Nemoto K, Tezuka Y, Sato T, Ikeda Y, Kobori M, Kawashima H, Eguchi H, Hakeda Y and Kumegawa M: Identification of matrix metalloproteinase 9 in rabbit osteoclasts. J Biol Chem 269: 15006-15009, 1994.

42. Oka Y, Iwai S, Amano H, Irie Y, Yatomi K, Ryu K, Yamada S, Inagaki $\mathrm{K}$ and Oguchi $\mathrm{K}$ : Tea polyphenols inhibit rat osteoclast formation and differentiation. J Pharmacol Sci 118: 55-64, 2012.

43. Logar DB, Komadina R, Prezelj J, Ostanek B, Trost Z and Marc J: Expression of bone resorption genes in osteoarthritis and in osteoporosis. J Bone Miner Metab 25: 219-225, 2007.

44. Roberts HC, Knott L, Avery NC, Cox TM, Evans MJ and Hayman AR: Altered collagen in tartrate-resistant acid phosphatase (TRAP)-deficient mice: A role for TRAP in bone collagen metabolism. Calcif Tissue Int 80: 400-410, 2007.
45. Wilson SR, Peters C, Saftig P and Brömme D: Cathepsin K activity-dependent regulation of osteoclast actin ring formation and bone resorption. J Biol Chem 284: 2584-2592, 2009.

46. Lange AW and Yutzey KE: NFATc1 expression in the developing heart valves is responsive to the RANKL pathway and is required for endocardial expression of cathepsin K. Dev Biol 292: 407-417, 2006.

47. Jordán MJ, Moñino MI, Martínez C, Lafuente A and Sotomayor JA: Introduction of distillate rosemary leaves into the diet of the Murciano-Granadina goat: Transfer of polyphenolic compounds to goats' milk and the plasma of suckling goat kids. J Agric Food Chem 58: 8265-8270, 2010.

48. Nurmi A, Nurmi T, Mursu J, Hiltunen R and Voutilainen S: Ingestion of oregano extract increases excretion of urinary phenolic metabolites in humans. J Agric Food Chem 54: 6916-6923, 2006.

49. Konishi Y, Hitomi Y, Yoshida M and Yoshioka E: Pharmacokinetic study of caffeic and rosmarinic acids in rats after oral administration. J Agric Food Chem 53: 4740-4746, 2005.

50. Schindler G, Patzak U, Brinkhaus B, von Niecieck A, Wittig J, Krähmer N, Glöckl I and Veit M: Urinary excretion and metabolism of arbutin after oral administration of Arctostaphylos uvae ursi extract as film-coated tablets and aqueous solution in healthy humans. J Clin Pharmacol 42: 920-927, 2002. 